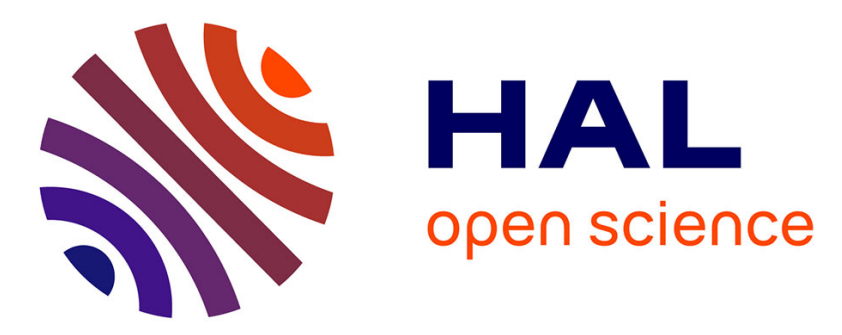

\title{
A Generic Model for Power Park Modules for Both Transient and Small-signal Stability Analysis
}

\author{
M. Belhocine, B. Marinescu, F Xavier
}

\section{To cite this version:}

M. Belhocine, B. Marinescu, F Xavier. A Generic Model for Power Park Modules for Both Transient and Small-signal Stability Analysis. PSCC, Jun 2018, Dublin, Ireland. hal-02516432

\section{HAL Id: hal-02516432 \\ https://hal.science/hal-02516432}

Submitted on 23 Mar 2020

HAL is a multi-disciplinary open access archive for the deposit and dissemination of scientific research documents, whether they are published or not. The documents may come from teaching and research institutions in France or abroad, or from public or private research centers.
L'archive ouverte pluridisciplinaire HAL, est destinée au dépôt et à la diffusion de documents scientifiques de niveau recherche, publiés ou non, émanant des établissements d'enseignement et de recherche français ou étrangers, des laboratoires publics ou privés. 


\section{A Generic Model for Power Park Modules for Both Transient and Small-signal Stability Analysis}

\author{
M. Belhocine \\ Ecole Centrale Nantes-LS2N-CNRS \\ Nantes, France \\ mohamed.belhocine@ec-nantes.fr
}

\author{
B. Marinescu \\ Ecole Centrale Nantes-LS2N-CNRS \\ Nantes, France \\ bogdan.marinescu@ec-nantes.fr
}

\author{
F. Xavier \\ RTE R\&D Division \\ Versailles, France \\ florent.xavier@rte-france.com
}

\begin{abstract}
Power Park Modules (PPM), such as wind and photovoltaic plants, bring new modelling challenges and they are usually aggregated into equivalent or generic models for stability analysis. However, most of the existing approaches to build these models are suited only for the transient stability and, generally, for a specific kind of PPM like wind farms. Moreover, some of them might not be always appropriate in the case of large-scale systems since they require significant computational effort. In this paper, a new methodology is proposed to construct generic models for PPM based on transfer matrices. It is suited for both transient and small-signal stability analysis, independently on the kind of the PPM and its technology. It has also low computational requirements. Its validation is done in Matlab and Eurostag software's by considering a realistic power system of 23 generators to which a wind farm is connected.
\end{abstract}

Index Terms-curve fitting, power park modules, power systems.

\section{INTRODUCTION}

In modern power systems, the amount of the electric power generated by Power Parc Modules (PPM), i.e., renewable energies like wind turbines and solar photovoltaic (PV) plants, connected to the grid by power electronics, increases significantly each year. As a consequence, its impact on the behaviour of the overall power system and, especially, on its stability becomes important and has to be taken into account in dynamic security assessment. Generally, this cannot be done by considering the detailed model of each device (e.g., wind turbine) due to their large number and distributed locations. Moreover, the TSO (Transmission System Operators) may have limited access to such detailed models and their parameters. This is why different techniques were proposed in literature (see, e.g., [1], [2], [3], [4]) to construct equivalent models for these new electric power generations.

Mainly there are two families of equivalent models. The ones based on physical representation, like an equivalent machine, and the ones with less physical meaning based on transfer functions. Both are used to reproduce the behaviour of active and reactive powers injected by the PPM into the grid, but the second ones are more attractrive when only few data are available on the physical structure of the PPM, its control system and the used technologies. Indeed, since they are just mathematical models, they are better suited to build generic models in different situations, i.e., they are flexible models. This is illustrated, e.g., in [1] and [2] where such generic models were built for wind farms. However this flexibility was not sufficiently exploited in order to make the generic models suited for several applications. For instance, most of the proposed approaches allow constructing equivalent models only for the transient stability and, generally, for a specific kind of PPM like wind farms. This is obviously important but not sufficient to fully analyse the stability of the system which is usually evaluated at two levels: the transient stability in case of large disturbances like a fault and the small-signal stability in case of small disturbances for which the behaviour of the system can be considered linear. Both are related in the sense that the small-signal stability can be analysed by linearising, around a steady state point, the dynamic model used to analyse the transient stability. This gives informations on how the modes of the system change after connecting the PPM to the grid. Especially, the electromechanical modes (see, e.g., [5]) or, more generally, coupling modes which involve distant grid devices [6], [7]. As a consequence, the target of the generic model of the PPM is to capture the information related to both kinds of phenomena (transient and small-signal). Indeed, if the latter is built only for one class of phenomena, there is no guarantee that the other one will be well reproduced. This situation has been put into evidence in [?] where the impact of a model of wind generation on the inter-area modes was studied.

Several techniques were proposed to tune the parameters of the equivalent models. For example, the ones (used, e.g., in [2]) in which the full dynamic model of the grid is simulated at each step time in order to update the parameters of the equivalent model. This has the advantage to give accurate results since the behaviour of the injected powers is adjusted at each iteration based on a feedback from the simulation of the actual model of the grid. However, when the latter is too complex and of large-scale, this way of doing might require significant computational effort.

Here, we propose a methodology to construct a unique PPM generic model suited for the aforementioned target phenomena with low computational effort. The idea is similar to the one proposed in [1] which consists of using an input-output transfer matrix to reproduce the behaviour of active and reactive powers injected into the grid when the system is disturbed by a set of faults. The difference here is that the methodological framework is extended to consider different 
kinds of sources (wind, solar, etc.) independently of their technology, to take into account the small-signal stability and to tune the parameters without using the model of the grid. A structure is first proposed for the generic model. Next, to capture informations related to the target phenomena, time and frequency domains curve fitting criteria are introduced and combined into the same cost function. The resulting optimization problem is solved using only the structure of the generic model and some recorded signals from the full model in which the PPM is fully represented.

The paper is organized as follows: in Section II, the methodology is given. Section III explains the tuning of the parameters of the generic model. The test system and the obtained results are shown in Section IV while Section V is devoted to conclusions.

\section{Methodology AND hypothesis}

In a power system, each PPM (with one or more units) is connected to a single connection node of the grid via power electronic devices like back-to-back structures. Thus, both grid and PPM (with its connexion device) exchange active and reactive powers during time and they form two interconnected subsystems as shown in Fig. 1a.

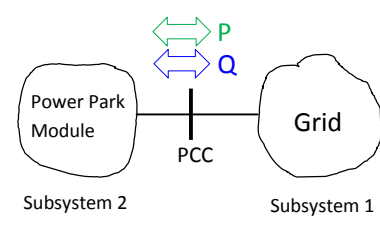

(a) Connexion of a PPM to the grid

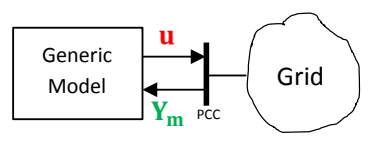

(b) Concept of generic model
Figure 1. Physical and generic models of PPM

Based on these considerations, the idea behind a generic model is to represent the full physical and detailed model of the PPM by a simpler one (as shown in Fig. 1b) in which the model of subsystem 2 does not have necessarily a physical meaning. Its main role is to reproduce the behaviour of active and reactive powers injected by the PPM into the grid when the whole interconnected system is disturbed, e.g., by a grid fault. For this, local signals like the voltage at the connexion node, injected current, exchanged powers can be used as inputs and outputs of the generic model. Indeed, $\mathbf{Y}_{\mathbf{m}}(t)$ and $\mathbf{u}(t)$, shown in Fig. 1b, are not necessarily the inputs and the outputs of the physical model of the PPM since, in a realistic situation, it can be not easy to identify them. For instance, if the PPM is connected to the grid by a transmission line, it is clear that the current through the line and the voltage at the Point of Common Coupling (PCC) are the interconnection variables. However, it is not clear which of them is the input or the output of the PPM. This is why hypothesis are generally made on the structure of the generic model as well as on its input and output signals. In our case, the way in which this is done is explained below along with the basic idea of the proposed methodology.

\section{A. Basic idea}

To reproduce the target phenomena, informations related to them are firstly needed. They are used to tune the parameters of the generic model. Based on this, the idea here is to use both time and frequency responses of the system. More precisely, to capture the transient stability, the proposed strategy is to disturb the system by a set of faults $\mathcal{N}_{F}$ at critical nodes. The latter are chosen such that (i) they have the Critical Clearing Time (CCT) smaller than the others, (ii) they cover well the zone around the PCC. Condition (i) is to capture the transient stability while condition (ii) is to give acceptable results for other sets of faults. The approach is a little different for the small-signal phenomenon since, in this case, the reference is a Bode diagram. More specifically, as the modes of the system can be captured by its linearized model around a steady-state point, the parameters of the generic model can be tuned to fit the Bode diagrams of a well-chosen input-output transfer issued from the full detailed model of the power system. An overview on the complete methodology including all these steps is given in the diagram of Fig. 2.

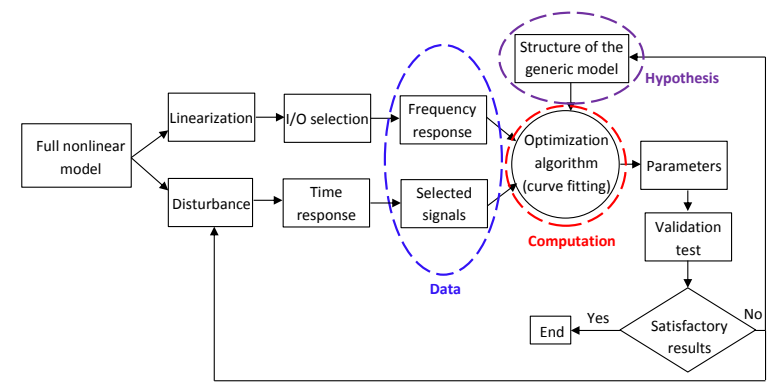

Figure 2. Steps of the proposed methodology

\section{B. Structure of the generic model}

As mentioned before, the structure of the generic model is not unique and it can be chosen in function of the objectives. In our case, the structure proposed for the generic model is shown in Fig. 3.

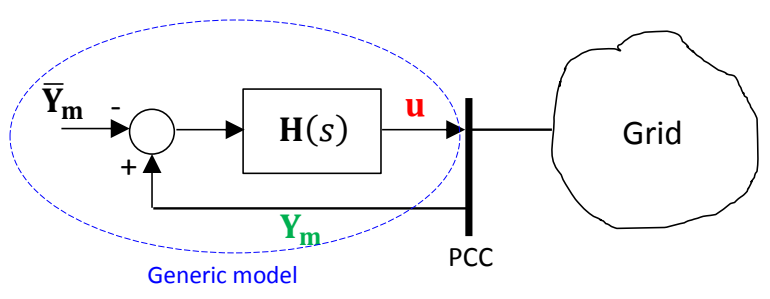

Figure 3. Integration scheme of the generic model

It consists of a multivariable transfer matrix $\mathbf{H}(s)$ connected to the model of the grid by a feedback loop in which $\overline{\mathbf{Y}}_{\mathbf{m}}$ is the steady state value of $\mathbf{Y}_{\mathbf{m}}(t)$. This choice is mainly motivated by two reasons. First, by the need to develop a unique generic model able to reproduce the aforementioned target phenomena. Indeed, such a structure is more convenient to take into account the small-signal phenomena in the sense that it is 
already linear and does not need to be linearized. Next, it can be used to capture the power injected by different kinds of sources independently on their technologies. As shown in Section III, only some measured signals are needed to tune the parameters of the transfer matrix $\mathbf{H}(s)$. The latter has the following form

$$
\mathbf{H}(s)=\left[\begin{array}{ccc}
H_{11}(s) & \cdots & H_{1 p}(s) \\
\vdots & & \vdots \\
H_{q 1}(s) & \cdots & H_{q p}(s)
\end{array}\right],
$$

in which $p$ and $q$ are, respectively, the number of inputs and outputs. For each $H_{i j}(s)$, the assumed form is as follows

$$
H_{i j}(s)=\frac{\sum_{k=0}^{m_{i j}} a_{k}^{(i, j)} s^{k}}{\sum_{\ell=0}^{n_{i j}-1} b_{\ell}^{(i, j)} s^{\ell}+s^{n_{i j}}}
$$

where $m_{i j}$ and $n_{i j}$, with $(i, j) \in\{1, \cdots, p\} \times\{1, \cdots, q\}$, are, respectively, the numerator and denominator degrees of $H_{i j}(s)$. All the coefficients $a_{k}^{(i, j)} \in \mathbb{R}^{m_{i j}+1}$ and $b_{\ell}^{(i, j)} \in \mathbb{R}^{n_{i j}}$ are then to be tuned together in order to reproduce the target phenomena. In the sequel, all the coefficients of $\mathbf{H}(s)$ are grouped into the following vector

$$
\Theta=\left[\left\{a_{k}^{(i, j)}\right\}_{k}^{i, j}\left\{b_{\ell}^{(i, j)}\right\}_{\ell}^{i, j}\right],
$$

called here vector of parameters. Finally, only the input $\mathbf{u}(t)$ and the output $\mathbf{Y}_{\mathbf{m}}(t)$ of the generic model have to be fixed. For this, one assumes here that the generic model of Fig. 1b is a dynamic power or current injector, i.e., the output $\mathbf{u}(t)$ is

$$
\mathbf{u}(t)=\left[\begin{array}{l}
P(t) \\
Q(t)
\end{array}\right] \text { or } \mathbf{u}(t)=\left[\begin{array}{c}
\mathrm{I}_{\mathrm{R}}(t) \\
\mathrm{I}_{\mathrm{I}}(t)
\end{array}\right],
$$

where $P(t), Q(t)$ are the injected active and reactive powers, respectively, while $\mathrm{I}_{\mathrm{R}}(t)$ and $\mathrm{I}_{\mathrm{I}}(t)$ are the real and the imaginary parts of the injected current (if system equations are written in a grid (load-flow) axes). That is, in both cases, the following relationships

$$
\left\{\begin{array}{l}
P(t)=\mathrm{V}_{\mathrm{R}}(t) \mathrm{I}_{\mathrm{R}}(t)+\mathrm{V}_{\mathrm{I}}(t) \mathrm{I}_{\mathrm{I}}(t) \\
Q(t)=\mathrm{V}_{\mathrm{R}}(t) \mathrm{I}_{\mathrm{I}}(t)-\mathrm{V}_{\mathrm{I}}(t) \mathrm{I}_{\mathrm{R}}(t)
\end{array},\right.
$$

at the connection node PCC, are satisfied where $\mathrm{V}_{\mathrm{R}}(t)$ and $\mathrm{V}_{\mathrm{I}}(t)$ are the real and the imaginary parts of the voltage $V_{\text {PCC }}(t)$ at the node PCC. Notice, however, that for both kinds of injectors (power or current), the injected variable is always a current. Indeed, if $\mathrm{V}_{\mathrm{R}}(t)$ and $\mathrm{V}_{\mathrm{I}}(t)$ are fixed by the voltage at the node to which the injector is connected (here PCC), equation (4) shows that two variables are free. It is then possible to inject a desired current $I_{d}(t)=\left(\mathrm{I}_{\mathrm{R}_{d}}(t), \mathrm{I}_{\mathrm{I}_{d}}(t)\right)$ or inject $\mathrm{I}_{\mathrm{R}}(t)$ and $\mathrm{I}_{\mathrm{I}}(t)$ to have desired trajectories for $P(t)$ and $Q(t)$.

Thus, when the kind of the injector is selected, the feedback signal $\mathbf{Y}_{\mathbf{m}}(t)$ can be chosen in order to close the loop. In the next section, more details and explanations are given on how all these different signals are used to tune the parameters of the generic model (1).

\section{PARAMETERS TUNING PROCEDURE}

Before explaining how to tune the parameters of the generic model (1), let us first recall that our goal is to find a unique and optimal vector of parameters $\Theta^{\text {opt }}$ which can lead to satisfy all the fixed objectives. For this, the latter have to be considered all together by formulating each of them. The way in which this is done here is presented and explained in what follows.

\section{A. Transient stability index}

Usually, to reproduce the transient stability phenomenon, the standard technique of least squares optimization is used to tune the parameters of the equivalent model. However, since this is often done by optimization interwoven with simulations, an important computation effort may be needed. For instance, if one considers the structure of Fig. 3, such an approach consists of minimising simulating the closed-loop system (i.e., with the model of the grid) and update the parameters of the vector $\Theta$ at each simulation step time in order to match the signal $\mathbf{u}$ with the one recorded or measured from the original full model of the power system. This can be easy when the model of the grid is very simple, but for complex and largescale models, such a technique becomes less feasible. This motivated us to propose a non imbricated tuning procedure which can be explained based on the diagram of Fig. 4.

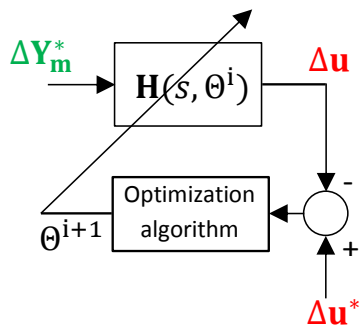

Figure 4. Non imbricated tuning procedure

To start, let us first denote by $\mathbf{u}^{*}(t)=\overline{\mathbf{u}}+\Delta \mathbf{u}^{*}(t)$ and $\mathbf{Y}_{\mathbf{m}}^{*}=\overline{\mathbf{Y}}_{\mathbf{m}}+\Delta \mathbf{Y}_{\mathbf{m}}^{*}(t)$ where $\overline{\mathbf{u}}$ and $\overline{\mathbf{Y}}_{\mathbf{m}}$ are the steady state values, the signals recorded from the simulation of the full model when its is disturbed by a set of faults at the critical nodes (see Section II-A). This implies that if $\mathbf{u}^{*}(t)$ is as defined by (3), i.e., powers or currents, then when $\mathbf{u}(t)=\mathbf{u}^{*}(t)$ in the diagram of Fig. 1b, we will obtain $\mathbf{Y}_{\mathbf{m}}(t)=\mathbf{Y}_{\mathbf{m}}^{*}(t)$. Based on this, the role of the generic model is then to inject a signal $\mathbf{u}$ as close as possible to $\mathbf{u}^{*}$ when the system of Fig. 3 is disturbed in the same way as the original one of Fig. 1a. For this, one has to find the optimal parameters $\Theta^{*}$ for $\mathbf{H}(s, \Theta)$ which make the square of the error between $\Delta \mathbf{u}(t)$ and $\Delta \mathbf{u}^{*}(t)$ as small as possible (Fig. 4). Mathematically, this can be formulated in two steps. First, define the following optimisation index

$$
J_{T}(\Theta)=\sum_{t_{k} \in \mathcal{T}}\left\|\Delta \mathbf{u}^{*}\left(t_{k}\right)-\Delta \mathbf{u}\left(t_{k}, \Theta\right)\right\|_{2}^{2},
$$

where $\|\cdot\|_{2}$ is the usual 2 -norm and $\mathcal{T}=\left\{t_{0}, t_{1}, \cdots, T\right\}$ is the time window (of size $T$ ) in which the signal $\mathbf{u}^{*}$ is available. Next, solve the associated optimization problem given by 


$$
\Theta^{*}=\underset{\Theta \in \mathbb{R}^{n}}{\operatorname{argmin}} J_{T}(\Theta)
$$

where $n=\left(\sum_{i=1}^{q} \sum_{j=1}^{p} n_{i j}+m_{i j}\right)+p q$.

Notice that, in this case, it is not needed to add stability constraints to (5) since if one has a good fitting (i.e., $\mathbf{u}$ close to $\mathbf{u}^{*}$ ), the stability of the closed-loop system of Fig. 3 can be preserved and all the poles of $\mathbf{H}\left(s, \Theta^{*}\right)$ are with real part less or equal to zero. However, the situation is not the same for the small-signal stability as one can see in the next section.

\section{B. Small-signal stability index}

For the power system of Fig. 1a, it is interesting to know how the modes change when connecting the PPM to the grid. Especially, the modes of interest which are mainly the electromechanical modes like the inter-area ones and coupling modes in general. The latter were introduced in [6] and [7] as modes involving distant devices and are not necessarily of electromechanical nature. In [8], e.g., a sensitivity index is introduced to analyse the impact of a wind farm on these modes based on its equivalent model which can be e.g., the generic one that we propose. This is why it is important that the generic model reproduces as well as possible such modes in the closed-loop system of Fig. 3. For this, a curve fitting in the frequency domain is proposed here. More precisely, after linearising the dynamic model of the system of Fig. 1a, each input-output transfer can captures the modes of the system which are dominant in that transfer. Notice that the ones which are not dominant are not of interest for our problem since they are not impacted by the considered PPM injection. Moreover, the most impacted modes by the power injection are, generally, related to the devices located near the injection point. Thus, by selecting a frequency range to which belong the modes of interest, any transfer (possibly multivariable) of the system related to a sensitive device to PPM injection and in which these modes are dominant can be chosen. Its Bode diagram plays the role of a reference which has to be reproduced when the generic model is used.

To formulate this, let us start by the diagram of Fig. 5 which is the small-signal stability representation of the system of Fig. 3, where $\mathbf{G}(s)$ is the transfer matrix of the grid model linearised around the equilibrium point. The variables $\mathbf{W}$ and $\mathbf{Z}$ are respectively the inputs and the outputs of the chosen transfer in which the modes of interest are dominant and sensitive to PPM injection.

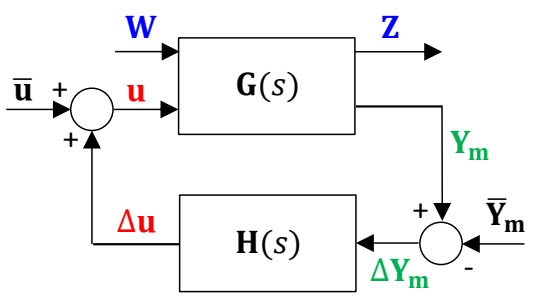

Figure 5. Closed-loop system for the small-signal stability
From the diagram of Fig. 5, the transfer between $\mathbf{W}$ and $\mathbf{Z}$ can be written by the following linear fractional transformation (see, e.g., [9])

$$
\mathcal{F}_{l}(\mathbf{G}, \mathbf{H}(\Theta))=\mathbf{G}_{11}+\mathbf{G}_{12}\left[I-\mathbf{H}(\Theta) \mathbf{G}_{22}\right]^{-1} \mathbf{H}(\Theta) \mathbf{G}_{21}
$$

which is the closed-loop transfer matrix, with

$$
\left[\begin{array}{c}
\mathbf{Z} \\
\mathbf{Y}_{\mathbf{m}}
\end{array}\right]=\underbrace{\left[\begin{array}{ll}
\mathbf{G}_{11} & \mathbf{G}_{12} \\
\mathbf{G}_{21} & \mathbf{G}_{22}
\end{array}\right]}_{\mathbf{G}}\left[\begin{array}{l}
\mathbf{W} \\
\mathbf{u}
\end{array}\right] .
$$

Now, let us consider that $\omega^{+}$and $\omega^{-}$are, respectively, the upper and the lower frequency bands of the frequency range $\Omega^{\star}=\left[\omega^{+} \omega^{-}\right]$to which belong the frequencies corresponding to the modes of interest. More precisely, if one denotes by $\Lambda$ the set of all the modes of the linearised model of the system of Fig. 1a, the set of modes of interest $\Lambda^{\star} \subset \Lambda$ is such that

$$
\Lambda^{\star}:=\left\{\lambda \in \Lambda \mid \operatorname{Im}(\lambda) \in \Omega^{\star}\right\} .
$$

Let also $\mathbf{F}^{\star}\left(i \omega_{k}\right) \in \mathbb{C}^{n_{z} \times n_{w}}$ with $\omega_{k} \in \Omega^{\star}, i^{2}=-1$ and $n_{z}, n_{w}$ the length of $\mathbf{Z}$ and $\mathbf{W}$ respectively, be the frequency response, in the restricted frequency range $\Omega^{\star}$, of the transfer between $\mathbf{W}$ and $\mathbf{Z}$ selected from the linearised model of the initial system of Fig. 1a. It is then our reference which has to be fitted, in the frequency domain, by the closed-loop transfer matrix (6) in order to reproduce the modes of $\Lambda^{\star}$. For this, the index to be minimized is chosen

$$
J_{\Omega^{\star}}(\Theta)=\sum_{\omega_{k} \in \Omega^{\star}}\left\|\mathbf{F}^{\star}\left(i \omega_{k}\right)-\mathcal{F}_{l}\left(\mathbf{G}\left(i \omega_{k}\right), \mathbf{H}\left(i \omega_{k}, \Theta\right)\right)\right\|_{2}^{2}
$$

and the optimization problem to solve is

$$
\hat{\Theta}=\underset{\Theta \in \mathbb{R}^{n} \cap \mathcal{S}_{c}}{\operatorname{argmin}} J_{\Omega^{\star}}(\Theta),
$$

where $\mathcal{S}_{c}$ is the constraints domain, i.e., such that the poles of each $H_{i j}(s)$ are all of real part less or equal to zero. Indeed, here, one has to add at least stability constraints on the entries of $\mathbf{H}(s)$ since one could obtain a good frequency fitting with an unstable generic model. It is also possible to add constraints on the stability of the closed-loop transfer matrix (6) in order to ensure the stability of the system of Fig. 5.

Remark 1: For large-scale power systems, the input data $\mathbf{F}^{\star}\left(i \omega_{k}\right)$ and $\mathbf{G}\left(i \omega_{k}\right)$ with $\omega_{k} \in \Omega^{\star}$, can be obtained by using appropriate tools. In [10], for example, such data were obtained in the case of the European system for which the number of the state variables is about 20000. Likewise, to obtain the time domain trajectories, needed to tune the parameters of the generic model, some simulation tools like the Eurostag program [11], used here, are very efficient to simulate the behaviour of complex and large-scale power systems.

\section{Mixed index}

After giving the indexes corresponding to each kind of stability, one has now to mix them into a same objective function in order to find the optimal parameters $\Theta^{\text {opt }}$ which 
can lead the generic model to reproduce both small-signal and transient stabilities. This is very important since if a generic model is tuned only for one kind of stability, there is no guarantee that it can reproduce the other one. Such an objective function is chosen here as

$$
J_{T \Omega^{\star}}(\Theta)=\alpha J_{T}(\Theta)+\beta J_{\Omega^{\star}}(\Theta),
$$

where $\alpha$ and $\beta$ are two positive constants to give high or low priority to a kind of stability compared to the other one. Moreover, they can be scaled to belong to a set $\mathcal{D}$ defined by

$$
\mathcal{D}:=\left\{(\bar{\alpha}, \bar{\beta}) \in\left[\begin{array}{ll}
0 & 1
\end{array}\right] \times\left[\begin{array}{ll}
0 & 1
\end{array}\right] \mid \bar{\alpha}+\bar{\beta}=1\right\} .
$$

In this way, they can be expressed by percentages. Finally, the vector $\Theta^{\text {opt }}$ of optimal parameters can be obtained by solving the optimization problem bellow

$$
\Theta^{\text {opt }}=\underset{\Theta \in \mathbb{R}^{n} \cap \mathcal{S}_{c}}{\operatorname{argmin}} J_{T \Omega^{\star}}(\Theta) .
$$

Remark 2: (9) is a non linear optimization problem which can be solved by different programs like the fmincon function of Matlab. However, since the index $J_{T \Omega^{\star}}(\Theta)$ is not a convex function, with respect to the parameters of $\Theta$, it is possible to get local minimums which may not lead to satisfactory results. Thus, it is better to start with an initial vector $\Theta^{\text {init }}$ which makes the entries of $\mathbf{H}\left(s, \Theta^{\text {init }}\right)$ all stable.

\section{POWER SYSTEM APPLICATION}

To test and to validate our approach, we considered a realistic power system represented in Fig. 6 .

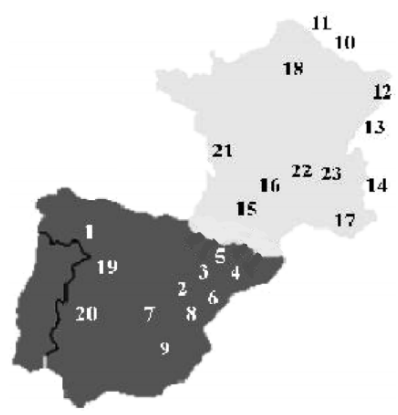

Figure 6. France-Span-Portugal interconnected system

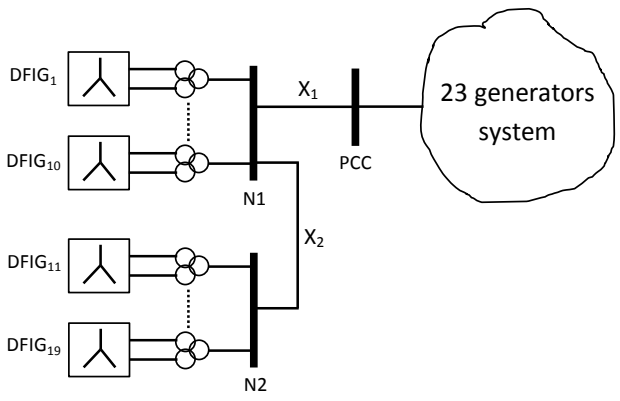

Figure 7. Test system
It is a simplified model of the France-Span-Portugal interconnection which consists of 23 synchronous generators to which is connected a wind farm of 19 DFIG (Double Fed Induction Generator) split into two groups as shown in Fig. 7. The DFIG's are firstly connected between them by a transmission line of reactance $\mathrm{X}_{2}$ which represents the geographic distance between them. Next, they are connected, via a transmission line of reactance $\mathrm{X}_{1}$, to $24 \mathrm{kV}$ terminal bus (Vandellos (PCC)) of Generator G8. The total active power generated by the wind farm is about $276 \mathrm{MW}$.

All the synchronous generators, as well as the DFIG's, are modelled in detail along with their different regulators. The resulting dynamic model has 1021 state variables among which more than half are related to the dynamic model of the wind farm which has to be replaced by a generic model.

Starting from this situation, the first step to design our generic model is to generate the input data, i.e., the reference trajectories for both transient and small-signal stabilities. This is done in two steps. First, the whole interconnected system is disturbed at time $100 \mathrm{~s}$ by a set of symmetrical faults (cleared after $200 \mathrm{~ms}$ ) at grid buses $\mathcal{N}_{F}=\{$ PCC, Begues (near G4), Foix (terminal of G6) $\}$ for which the CCT's are shown in the first line of Table I. Notice that $\mathcal{N}_{F}$ was chosen as explained in Section II-A.

TABLE I

CRITICAL ClEARING TIMES (IN MILLISECONDS)

\begin{tabular}{c|c|c|c|c}
\hline Node & PCC & Teruel & Begues & Foix \\
\hline With full model & 277 & 320 & 299 & 774 \\
\hline With generic model (case 1) & 272 & 313 & 310 & 768 \\
\hline With generic model (case 2) & 272 & 311 & 312 & 767 \\
\hline
\end{tabular}

This allowed us to get the trajectory of the voltage at node PCC, shown in Fig. 8, as well as the ones of the active (Fig. 9a) and the reactive (Fig. 9b) powers between nodes N1 and PCC, i.e., trough the transmission line of impedance $X_{1}$. Notice just that, to test the robustness of the proposed approach, terminal bus (Teruel) of generator G7 was chosen. It does not belong to set $\mathcal{N}_{F}$ and it has also a CCT smaller than the one of Foix.

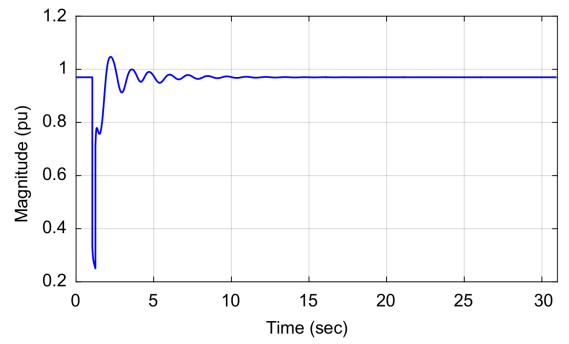

Figure 8. Response of $V_{\mathrm{PCC}}$ to a short-circuit at node PCC

Next, to capture all the coupling modes of the system, a frequency range between $\omega^{-}=0.01 \mathrm{rad} / \mathrm{s}$ (i.e., $0.0016 \mathrm{~Hz}$ ) and $\omega^{+}=30 \mathrm{rad} / \mathrm{s}$ (i.e., $4.77 \mathrm{~Hz}$ ) is fixed, i.e., $\Omega^{\star}=\left[\begin{array}{ll}0.01 & 30\end{array}\right]$ $\mathrm{rad} / \mathrm{s}$. Generator G8 is connected to PCC bus. Thus, according to the explanations of section III-B, the transfer between 
its mechanical torque and its speed is selected. Its Bode diagrams are shown in Fig. 10 and one can see that there are mainly three dominant mode. Especially, the inter-area one of frequency $0.8109 \mathrm{~Hz}$. Indeed, as mentioned, e.g., in [12], a dominant pole is the one for which the frequency is close to the frequency at which arises the peak in the Bode magnitude diagram.

Also, by choosing the generic model as a dynamic power injector, i.e., $\mathbf{u}=\{P(t), Q(t)\}$ for which $\mathbf{Y}_{\mathbf{m}}(t)=\left\{V_{\mathrm{PCC}}\right\}$, the frequency responses of all the entries of the transfer matrix $\mathbf{G}(s)$ are computed in the frequency range $\Omega^{\star}$. All these choices was motivates by practical reasons.

From all these data, two cases are considered. On one hand, the case where the parameters of the generic model are tuned to reproduce only one class of phenomena (transient), i.e., $\beta=0$ in (8). On the other hand, the case where the other class (small-signal) is taken into account with $\alpha=0.01$ and $\beta=0.99$. In both cases, the generic model is assumed of order 5, i.e., $n_{11}=m_{11}=n_{21}=m_{21}=5$ since from Figs. $9 \mathrm{a}$ and $9 \mathrm{~b}$ one can notice that there are at least two oscillatory modes. The optimization problem (8) is solved with fmincon function of Matlab and two generic models of the form

$$
\left[\begin{array}{c}
\Delta P \\
\Delta Q
\end{array}\right]=\underbrace{\left[\begin{array}{c}
H_{11}(s) \\
H_{21}(s)
\end{array}\right]}_{\mathbf{H}(s)} \Delta V_{\mathrm{PCC}},
$$

are obtained where the transfer matrix $\mathbf{H}(s)$ is given by

$$
\mathbf{H}_{1}(s)=\left[\begin{array}{c}
-\frac{220.6+236.9 s+189.3 s^{2}+85.65 s^{3}+11.63 s^{4}+2.195 s^{5}}{45.51+126.6 s+33.05 s^{2}+32.39 s^{3}+1.423 s^{4}+s^{5}} \\
\frac{-0.9334+43.25 s+80.4 s^{2}+107.6 s^{3}+3.851 s^{4}+2.827 s^{5}}{5.332+15.62 s+28 s^{2}+28.09 s^{3}+1.409 s^{4}+s^{5}}
\end{array}\right],
$$

for the first one and

$$
\mathbf{H}_{2}(s)=\left[\begin{array}{c}
-\frac{4.1349+1.3588 s+4.1466 s^{2}+0.814 s^{3}+0.5024 s^{4}+0.0045 s^{5}}{1+1.2143 s+1.1376 s^{2}+0.295 s^{3}+0.1444 s^{4}+0.0007 s^{5}} \\
\frac{-37.2+953.525 s+33.84 s^{2}+168.93 s^{3}+0.2533 s^{4}+3.6686 s^{5}}{80.94+193.63 s+54.68 s^{2}+35.76 s^{3}+2.3368 s^{4}+s^{5}}
\end{array}\right]
$$
西

for the second one. The results of the curve fitting obtained with both generic models are shown in Figs. 9 and 10.

For the transient stability, one can see in Figs. 9a and 9b that the output of the first generic model of order 5 fits very well the reference trajectories corresponding to the change of active and reactive powers. In the second case, i.e., when $\beta \neq 0$, the fitting of active power, shown in Fig. 9c, is less accurate than in the first case, but better results are obtained for the small-signal stability as one can see in Fig. 10. Indeed, the latter shows that the pic around $0.81 \mathrm{~Hz}$, corresponding to an inter-area mode of the full system, is better reproduced with the second generic model than with the first one. This can be also seen in the results of Table II. At this stage, two conclusions come out. The first one is that when a generic model is built only for one class of phenomena (transient), there is no guarantee that it can gives good results for the other one (small-signal). The second one, is that there is a trade-off between these two objectives.
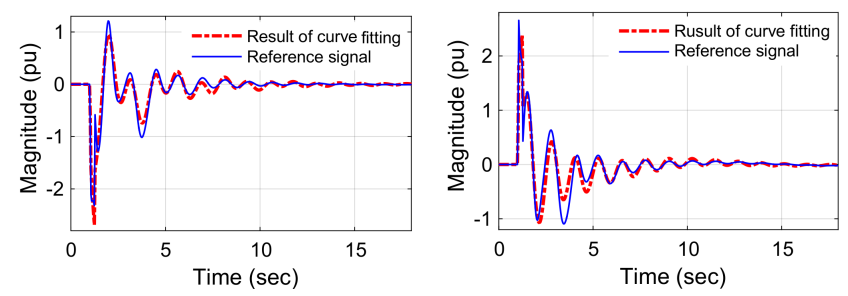

(a) Curve fitting result for active (b) Curve fitting result for reactive power (case 1)
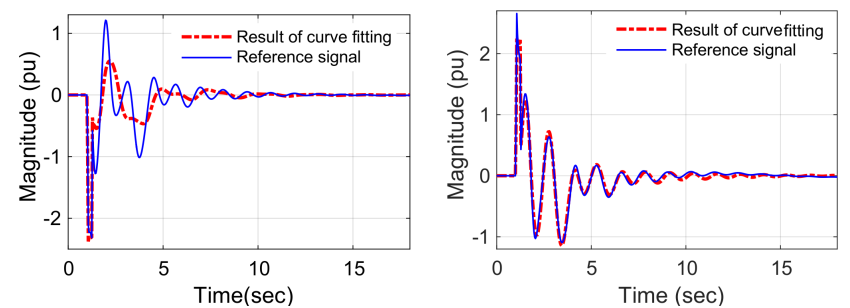

(c) Curve fitting result for active (d) Curve fitting result for reactive power (case 2)

power (case 2)

Figure 9. Curve fitting results for active and reactive powers
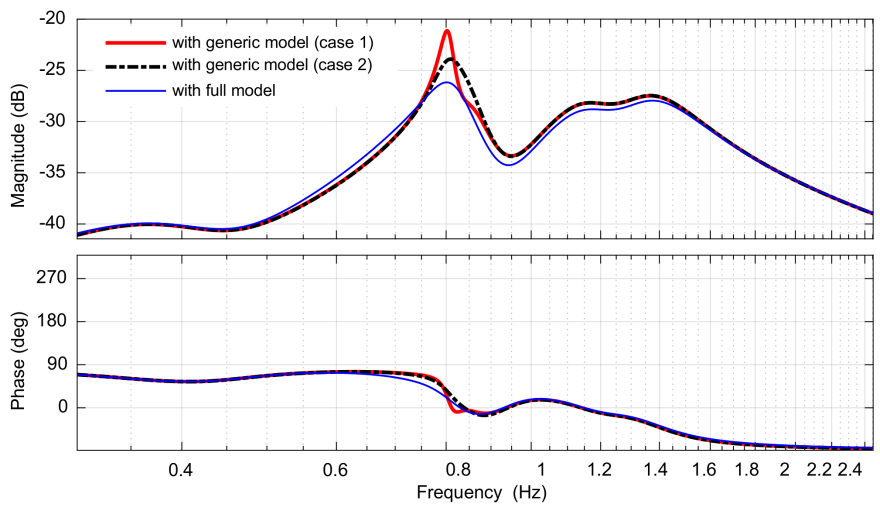

Figure 10. Frequency response fitting: reference and reproduced responses

Now, to validate our results, the obtained generic models have to be integrated to the nonlinear model of the grid as shown in Fig. 3. Indeed, Fig. 9 shows just the results of the curve fitting since, in our approach, the model of the grid is not used in the tuning procedure. For this, Eurostag program was used to construct the dynamic model corresponding to the system of Fig. 3 and to simulate its behaviour. A grid fault, started at $100 \mathrm{~s}$ and cleared after $200 \mathrm{~ms}$, is applied at the grid bus Teruel and the results are shown in Fig. 11.

As one can see in Figs., 11a and 11b, the first generic model (i.e., the one obtained with $\beta=0$ ) gives acceptable results for the transient stability (between $100 \mathrm{~s}$ and $103 \mathrm{~s}$ approximately). This shows the robustness of the proposed approach since, as mentioned before, a fault at bus Teruel was not considered when the reference trajectories, used in the tuning procedure, were generated. Second line of Table I claims also this since the CCT's computed with the generic model, at different grid buses, are not far from the ones 


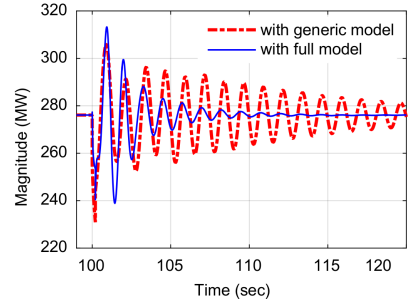

(a) Response of active power (case 1)

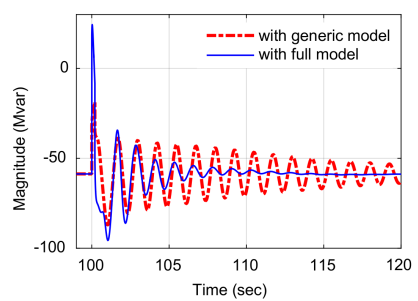

1)

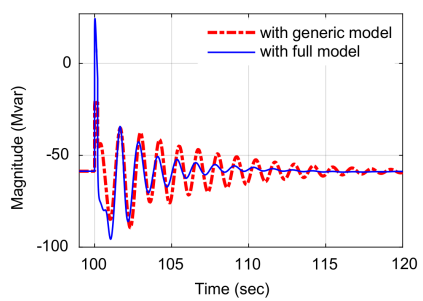

(c) Response of active power (case 2) (d) Response of reactive power (case 2)

Figure 11. Response of powers with both generic and full models

computed with the original full model. However, after 103 $\mathrm{s}$, one can notice that there are a lot of oscillations. The latter are related to the small-signal stability and they are dues to the pic (around $0.81 \mathrm{~Hz}$ ) in the Bode gain diagram of Fig. 10. Indeed, as the small-signal stability is not considered in this case, the resulting generic model disturbs a lot the interarea mode of frequency $0.8109 \mathrm{~Hz}$ by reducing its damping from $7.27 \%$ to $4.71 \%$ as shown in Table II. Notice that is other situations, the differences could be more important. For example, in [?], the inter-area mode of interest was instable with the generic model built only for transient stability.

In the second case, the situations is, however, more acceptable for the small-signal stability as one can see in Figs. 11c and $11 \mathrm{~d}$ but less accurate for the active power as shown in Fig. 11c. The reason is that, with the second generic model, the inter-area mode of frequency $0.8109 \mathrm{~Hz}$ is better reproduced than in the first case. Its damping (of $6.05 \%$ ) is more close to the one of the full model as shown in Table II. This can be also seen in Fig. 10 where the pic around $0.81 \mathrm{~Hz}$ is less important in the second case. However, as there is a trade-off between small-signal and transient stabilities, one can notice that the less accurate (but still acceptable since the CCT's are almost the same with the previous case) result obtained for the active power is coherent with the curve fitting result of Fig. 9a. A possible way to improve this result is to increase the order of the generic model (1).

\section{CONClusion}

An optimal and not interwoven with simulations methodology to build generic models for PPM, integrated into largescale power systems, is presented in this paper. Its advantages compared to the existing techniques are mainly:
TABLE II

FREQUENCY AND DAMPING OF INTER-AREA MODE

\begin{tabular}{c|c|c}
\hline & Frequency (Hz) & Damping (\%) \\
\hline With full model & 0.8109 & 7.27 \\
\hline With generic model (case 1) & 0.8134 & 4.71 \\
\hline With generic model (case 2) & 0.833 & 6.05 \\
\hline
\end{tabular}

- it allows to reproduce both kinds of phenomena (transient and small-signal) with the same generic model.

- it requires less computational effort since the parameters of the generic model are tuned without using the dynamic model of the grid.

- it is suited for different kinds of power sources independently on their technologies.

A realistic power system application is considered for which acceptable results are obtained. This motivate us to apply the proposed approach in the case of more complex systems like the European one. Its application to another kind of sources like PV plants will be also tested. In the case of several PPM, a coordinate tuning of parameters is under development. For the TSO, all the above advantages can be exploited, e.g., to improve numerical simulations, to better design voltage and power controllers and to exchange models between them in a more flexible way.

\section{REFERENCES}

[1] F. Wu, Y.-X. Chen, G.-J. Gong, and L. Shi, "Non-mechanism equivalent model of wind farm for transient stability analysis," in 5th International Conference on Electric Utility Deregulation and Restructuring and Power Technologies (DRPT). IEEE, 2015, pp. 183-186.

[2] I. Erlich, F. Shewarega, C. Feltes, F. Koch, and J. Fortmann, "Determination of dynamic wind farm equivalents using heuristic optimization," in IEEE PES General Meeting. IEEE, 2012, pp. 1-8.

[3] D. Remon, A. M. Cantarellas, and P. Rodriguez, "Equivalent model of large-scale synchronous photovoltaic power plants," IEEE Transactions on Industry Applications, vol. 52, no. 6, pp. 5029-5040, 2016.

[4] P. Pourbeik, J. Sanchez-Gasca, J. Senthil, J. Weber, P. Zadehkhost, Y. Kazachkov, S. Tacke, J. Wen, and A. Ellis, "Generic dynamic models for modeling wind power plants and other renewable technologies in large scale power system studies," IEEE Transactions on Energy Conversion, 2016 ('in press').

[5] G. Rogers, Power System Oscillations. Boston, USA: Kluwer Academic Publishers, 2000.

[6] L. Arioua and B. Marinescu, "Robust grid-oriented control of high voltage dc links embedded in an ac transmission system," International Journal of Robust and Nonlinear Control, vol. 26, no. 9, pp. 1944-1961, 2016.

[7] - "Multivariable control with grid objectives of an hvdc link embedded in a large-scale ac grid," International Journal of Electrical Power \& Energy Systems, vol. 72, pp. 99-108, 2015.

[8] W. Du, X. Chen, and H. Wang, "Impact of dynamic interactions introduced by the dfigs on power system electromechanical oscillation modes," IEEE Transactions on Power Systems, 2017.

[9] K. Zhou and J. C. Doyle, Essentials of robust control. Prentice hall Upper Saddle River, NJ, 1998, vol. 104.

[10] B. Marinescu and D. Petesch, "Three-level coordination in power system stabilization," Electric Power Systems Research, vol. 111, pp. 40-51, 2014.

[11] B. Meyer and M. Stubbe, "Eurostag, a single tool for power system simulation," Transmission \& Distribution International, pp. 47-52, 1992.

[12] W. H. A. Schilders, H. A. van der Vorst, and J. Rommes, Model Order Reduction: Theory, Research Aspects and Applications. Berlin, Germany: Springer-Verlag Berlin Heidelberg, 2008. 